\title{
FAMILIES OF REAL AND SYMMETRIC ANALYTIC FUNCTIONS
}

BY

\author{
YUSUF ABU-MUHANNA AND THOMAS H. MACGREGOR
}

\begin{abstract}
We introduce families of functions analytic in the unit disk and having rotational symmetries. The families include the $k$-fold symmetric univalent functions which have real coefficients. We relate the families to special classes of functions with a positive real part and then determine their extreme points. The case $k=2$ corresponds to the odd functions which "preserve quadrants" and the extreme points of this set are characterized by having a radial limit which is real or imaginary almost everywhere. We also find estimates on the initial coefficients of functions in the families.
\end{abstract}

1. Introduction. We shall study families of functions which are analytic in the open unit disk and have symmetries which may roughly be described as "preserving sectors". The families provide generalizations and specializations of the idea of a typically-real function. Our main concern is the determination of the extreme points of each family.

We now define one of these families, which is associated with the property of "preserving quadrants". It is a special case $(k=2)$ of what is introduced near the end of the paper. Throughout we let $\Delta=\{z:|z|<1\}$.

Definition. Let $Q$ denote the set of all functions $f$ that are analytic in $\Delta$, satisfy $f(0)=0$ and $f^{\prime}(0)=1$ and for which $f(z)$ is in the $j$ th (closed) quadrant whenever $z$ is in the $j$ th quadrant and $|z|<1(j=1,2,3,4)$.

The class $Q$ was introduced by $\mathbf{M}$. S. Robertson [7]. The condition that $f$ "preserves quadrants" implies that $f(z)$ is real when $z$ is real and $f$ is odd (as we verify later). It also implies that $f(0)=0$ and $f^{\prime}(0)>0$ so that the normalization $f^{\prime}(0)=1$ is not a real restriction.

The class $Q$ contains the subclass of normalized, odd univalent functions which have real coefficients. $Q$ also is contained in the class of odd typically-real functions. Let $T$ denote the set of typically-real functions introduced by W. Rogosinski in [8]. A function $f$ analytic in $\Delta$ belongs to $T$ if and only if $f(0)=0$, $f^{\prime}(0)=1$ and

$$
\operatorname{Im} z \cdot \operatorname{Im} f(z) \geqslant 0 \text { for }|z|<1 .
$$

The class $Q$ is characterized by condition (1) and

$$
\operatorname{Re} z \cdot \operatorname{Re} f(z) \geqslant 0 \text { for }|z|<1
$$

and the normalizations.

Received by the editors March 13, 1979 and, in revised form, December 10, 1979.

AMS (MOS) subject classifications (1970). Primary 30A76, 30A32; Secondary 30A42, 30A78.

Key words and phrases. Analytic function, typically-real function, extreme point, univalent function, functions with a positive real part, radial limit function, Poisson representation, harmonic measure, subordination, $k$-fold symmetric function, coefficient estimates. 
The set of extreme points of $T$ is given by

$$
f(z)=\frac{z}{(1-x z)(1-\bar{x} z)} \quad(|x|=1)
$$

as proved by L. Brickman, T. MacGregor and D. Wilken in [1]. The set of extreme points of the family of odd functions in $T$ was found by D. Hallenbeck in [4] and consists of the functions given by

$$
f(z)=\frac{z+z^{3}}{\left(1-x z^{2}\right)\left(1-\bar{x} z^{2}\right)} \quad(|x|=1) .
$$

We shall prove that the extreme points of $Q$ are characterized by the condition that $f\left(e^{i \theta}\right)$ is real or imaginary for almost all $\theta$ on $[0,2 \pi]$. In general, if $f \in Q$ then the radial limit function defined by

$$
f\left(e^{i \theta}\right)=\lim _{r \rightarrow 1} f\left(r e^{i \theta}\right)
$$

exists for almost all $\theta$ (as we verify later). The nature of the extreme points of $Q$ is, therefore, quite different from the results described by equations (3) and (4) for two related classes. It compares more closely with the results obtained by J. Milcetich in [5] about the family of functions whose range lies in a strip or in an angular region $\{w$ : $|\arg w|<\alpha(\pi / 2)\}$ where $0<\alpha<1$. One of the arguments we present is an extension of a technique introduced by Milcetich.

This paper is organized so that the bulk of the material is with reference to the class $Q$. In the process of determining the extreme points of $Q$ we prove several lemmas which have other applications. Later on we introduce the more general classes and determine their extreme points. The last section contains coefficient bounds for functions belonging to $Q$ and its generalizations.

The functions we study are related to functions which are subordinate to the map onto an angular region and are real on the real axis. In particular, functions with a positive real part with additional symmetries play an important role. Several results are used or developed which concern the theory of $H^{p}$-spaces, especially about radial limit functions and the Poisson representation formula. This formula and other facts relating to $H^{p}$-spaces may be found in [2]. One of the critical steps in this paper involves the construction of a suitable analytic function in terms of harmonic measure. The facts we use about harmonic measure may be found in [10].

2. Preliminaries. In this section we present initial facts about $Q$ and introduce families of functions needed in the later development.

Suppose that $f \in Q$ and

$$
f(z)=z+\sum_{n=2}^{\infty} a_{n} z^{n} \quad(|z|<1) .
$$

Since $f(z)$ is real when $z$ is real it follows that the coefficients $\left\{a_{n}\right\}$ are real. We now show that $f$ is odd. This depends on two reflections. Namely, since $f(z)$ is real when $z$ is real we conclude that $f(\bar{z})=\overline{f(z)}$. Also, since $f(z)$ is purely imaginary when $z$ is purely imaginary we find that $f(-\bar{z})=-\overline{f(z)}=-f(z)$. Therefore, $f(-z)=\overline{f(-\bar{z})}=$ $-f(z)$. 
Definition. Let $P$ denote the set of functions $p$ that are analytic in $\Delta$ and satisfy $p(0)=1$ and $\operatorname{Re} p(z)>0$ for $|z|<1$. Also, let $P_{R}$ denote the subset of $P$ so that $p \in P_{R}$ if $p(z)$ is real when $z$ is real (and $|z|<1$ ).

As was proved in [8] the mapping given by

$$
f(z)=z p(z) /\left(1-z^{2}\right) \quad(|z|<1)
$$

provides a one-to-one correspondence between $P_{R}$ and $T$. The following lemma establishes a similar characterization of $Q$ in terms of a special subset of $P_{R}$.

LemMa 1. A function $f$ belongs to $Q$ if and only if there is a function $p$ in $P_{R}$ so that

$$
f(z)=z p\left(z^{2}\right) /\left(1-z^{2}\right) \quad(|z|<1)
$$

and $p$ has the property that the function $q$ defined by

$$
q(z)=(1+z) p(z) /(1-z) \quad(|z|<1)
$$

also belongs to $P_{R}$.

Proof. Suppose that $f \in Q$. Let the function $g$ be defined by $g(z)=-i f(i z)$. From equation (2) we see that the conditions $\operatorname{Im} z \geqslant 0, \operatorname{Re} i z \leqslant 0, \operatorname{Re} f(i z) \leqslant 0$, $\operatorname{Im}[-i f(i z)] \geqslant 0$ are equivalent. Thus, $g \in T$. Consequently, because of equation (7), $g(z)=z q_{1}(z) /\left(1-z^{2}\right)$ where $q_{1} \in P_{R}$. Replacing $z$ by $-i z$ this relation becomes $f(z)=z q_{2}(z) /\left(1+z^{2}\right)$, where $q_{2}(z)=q_{1}(-i z)$ and thus $q_{2} \in P_{R}$. Since $f$ is odd, $q_{2}$ is even and therefore $q_{2}(z)=q\left(z^{2}\right)$ where $q \in P_{R}$. Summarizing these relations we find that

$$
f(z)=z q\left(z^{2}\right) /\left(1+z^{2}\right) \quad(|z|<1)
$$

where $q \in P_{R}$.

Similarly, since $f \in T$ and $f$ is odd we find that

$$
f(z)=z p\left(z^{2}\right) /\left(1-z^{2}\right),
$$

where $p \in P_{R}$. Equating the right-hand sides of equations (10) and (11) we find that the relation given in equation (9) holds for $p$ and $q$.

Conversely, suppose that there is a function $p$ in $P_{R}$ so that the function $q$ defined by equation (9) belongs to $P$ and let $f$ be defined by equation (8). Because the function $p_{1}$ defined by $p_{1}(z)=p\left(z^{2}\right)$ belongs to $P_{R}$, equation (8) immediately shows that $f \in T$, or, in other words, that equation (1) holds. The equations (8) and (9) imply equation (10). We conclude that $g \in T$, where $g(z)=-i f(i z)$, which is the same as condition (2). Therefore, $f \in Q$.

Definition. Let $P^{*}$ denote the set of functions $p$ so that $p \in P_{R}$ and the function $q$ defined by equation (9) also belongs to $P_{R}$.

The mapping given by equation (8) provides a linear homeomorphism between $P^{*}$ and $Q$. Therefore, the extreme points of the two convex sets are in one-to-one correspondence. We shall characterize $P^{*}$ in another way which leads to the determination of the extreme points of $P^{*}$.

In general, a function analytic in $\Delta$ and having a positive real part has a (finite) radial limit $p\left(e^{i \theta}\right)=\lim _{r \rightarrow 1} p\left(r e^{i \theta}\right)$ for almost all $\theta$ on $[0,2 \pi]$. Because of the correspondence given by equation (7), it follows that each function in $T$ also has a 
(finite) radial limit almost everywhere. This property holds, in particular, for $P_{R}$ and $Q$ and, in general, for functions in any subset of $P$ or $T$.

Definition. Let $P^{+}$denote the subset of $P$ consisting of the functions $q$ that satisfy

$$
\operatorname{Im} z \cdot \operatorname{Im} q(z) \geqslant 0 \text { for }|z|<1 .
$$

Except for the constant function $q=1, q$ is an open mapping. Thus, if $q \neq 1$ then $q \in P^{+}$if and only if $q(z)$ belongs to the (open) first quadrant when $\operatorname{Im} z>0$ (and $|z|<1$ ) and $q(z)$ belongs to the (open) fourth quadrant when $\operatorname{Im} z<0$ (and $|z|<1)$. If $q \in P^{+}$then $q(z)$ is real when $z$ is real and consequently the coefficients of the power series for $q$ are real. If $q \in P^{+}$and $q \neq 1$, then $q(z)=1+$ $q_{n} z^{n}+\ldots$ where $q_{n} \neq 0$. By considering the local behavior of $q$ at $z=0$ it is easy to show that $n=1$ and that $q_{1}>0$. Thus, except for $q=1$, if $q \in P^{+}$then $q^{\prime}(0)>0$.

Definition. Let $P^{-}$denote the subset of $P$ consisting of functions $p$ that satisfy

$$
\operatorname{Im} z \cdot \operatorname{Im} p(z)<0 \quad \text { for }|z|<1 .
$$

The family $P^{-}$is a subset of $P_{R}$, and, except for the function $p=1$, if $p \in P^{-}$ then $p^{\prime}(0)<0$. Note that the mapping defined by $p(z)=q(-z)$ gives a linear homeomorphism between $P^{-}$and $P^{+}$and, in general, provides information for one class from information about the other one.

3. The extreme points of $P^{*}, P^{+}$, and $P^{-}$. The following two lemmas shall be used to determine when a function $q$ belongs to our various classes in terms of the boundary value function $q\left(e^{i \theta}\right)$. Henceforth, we shall use the notation $m(A)$ to denote the Lebesgue measure of the set $A$ where $A$ is any subset of $[0,2 \pi]$.

LEMMA 2. Suppose that $q$ is a function which is analytic and nonvanishing in $\Delta$ and satisfies $|\arg q(z)|<\pi$ for $|z|<1$. Also, suppose that there is a subset $A$ of $[0,2 \pi]$ so that $m(A)=2 \pi$ and $q\left(e^{i \theta}\right)=\lim _{r \rightarrow 1} q\left(r e^{i \theta}\right)$ exists and satisfies $\operatorname{Re} q\left(e^{i \theta}\right)>0$ whenever $\theta \in A$. Then, $\operatorname{Re} q(z) \geqslant 0$ for $|z|<1$.

Proof. The function $s=q^{1 / 2}$ is analytic in $\Delta$ and satisfies $\operatorname{Re} s(z)>0$ for $|z|<1$, and thus the function $\psi=(s-1) /(s+1)$ is analytic in $\Delta$ and satisfies $|\psi(z)|<1$ for $|z|<1$. The function $\zeta=\left(w^{1 / 2}-1\right) /\left(w^{1 / 2}+1\right)$ maps the set $\{w$ : $\operatorname{Re} w \geqslant 0\} \cup\{\infty\}$ one-to-one onto a set $\Gamma$, which is a lens region symmetric about the real axis so that $\partial \Gamma$ consists of arcs of two circles passing through the points -1 and 1 . In particular, $\Gamma$ is a compact, convex subset of the closed unit disk.

Since $\operatorname{Re} q\left(e^{i \theta}\right) \geqslant 0$ when $\theta \in A$, it follows that $\psi\left(e^{i \theta}\right) \in \Gamma$ when $\theta \in A$. We may represent the bounded function $\psi$ as a Poisson integral of the function $\psi\left(e^{i \theta}\right)$. The function $\psi\left(e^{i \theta}\right)$ may be conveniently defined when $\theta \notin A$ as one of the values $\psi\left(e^{i \theta_{0}}\right)$ where $\theta_{0} \in A$. As $\Gamma$ is convex this implies that $\psi(z) \in \Gamma$ when $|z|<1$. We may assume that $\psi$ is not a constant function, and thereby conclude that $\psi(z)$ belongs to the interior of $\Gamma$ whenever $|z|<1$. Since the inerior of $\Gamma$ corresponds to the set $\{w: \operatorname{Re} w>0\}$ this shows that $\operatorname{Re} q(z)>0$ whenever $|z|<1$. 
LEMMA 3. Suppose that $q$ is a function analytic in $\Delta$ so that $q(0)=1,|\arg q(z)|<\pi$ for $|z|<1$ and $q(z)$ is real when $z$ is real $(|z|<1)$. Also, suppose that there is a subset $A$ of $[0,2 \pi]$ so that $m(A)=2 \pi$ and whenever $\theta \in A$ the following relations hold. $q\left(e^{i \theta}\right)=\lim _{r \rightarrow 1} q\left(r e^{i \theta}\right)$ exists, $\operatorname{Im} q\left(e^{i \theta}\right) \geqslant 0$ when $0 \leqslant \theta \leqslant \pi$ and $\operatorname{Im} q\left(e^{i \theta}\right)<$ 0 when $\pi \leqslant \theta \leqslant 2 \pi$. Then, $\operatorname{Im} z \cdot \operatorname{Im} q(z) \geqslant 0$ for $|z|<1$.

Proof. Let the functions $s, \psi$ and $\zeta$ be defined as in the proof of Lemma 2. Since the mapping defined between $w$ and $\zeta$ has the property $\operatorname{Im} \zeta \cdot \operatorname{Im} w>0$, the function $\psi$ satisfies $\psi(z)$ is real when $z$ is real $(|z|<1)$ and $\operatorname{Im} z \cdot \operatorname{Im} \psi(z)>0$ for almost all $\theta\left(z=e^{i \theta}\right)$. The condition $q(0)=1$ implies that $\psi(0)=0$ and thus the function $\omega$ defined by $\omega(z)=\left(1-z^{2}\right) \psi(z) / z$ is analytic in $\Delta$. Also, $\omega$ is bounded and $\operatorname{Re} \omega(z) \geqslant 0$ for almost all $\theta\left(z=e^{i \theta}\right)$. Appealing to the Poisson representation formula for $\omega$ we conclude that $\operatorname{Re} \omega(z) \geqslant 0$ for $|z|<1$. The lemma is certainly valid if $\psi$ is the constant function $\psi=0$ as this corresponds to $q=1$. Otherwise, we conclude that $\operatorname{Re} \omega(z)>0$ for $|z|<1$. In particular, $\psi$ can only have a simple zero at $z=0$. Thus, $\psi^{\prime}(0)$ is real and not zero. Since $\operatorname{Re} \psi^{\prime}(0)=\operatorname{Re} \omega(0)>0$ we conclude that $\psi^{\prime}(0)>0$.

The fact that $\psi^{\prime}(0)>0$ implies that if $\omega_{0}=\omega / \omega(0)$ and $\psi_{0}=\psi / \psi^{\prime}(0)$ then $\omega_{0} \in P_{R}$ and thus $\psi_{0} \in T$. In other words, $\operatorname{Im} z \cdot \operatorname{Im} \psi(z) \geqslant 0$ for $|z|<1$. Since the function $w=[(1+\zeta) /(1-\zeta)]^{2}$ maps the upper half of $\Delta$ onto the upper halfplane and the lower half of $\Delta$ onto the lower half-plane, this proves that $\operatorname{Im} z$. $\operatorname{Im} q(z) \geqslant 0$ for $|z|<1$.

Lemmas 2 and 3 are not valid without the assumption that $|\arg q(z)|<\pi$ for $|z|<1$. Any function that maps $\Delta$ one-to-one onto the complement of the slit $\{w: w=i y, y \leqslant 0\}$ satisfies $\operatorname{Re} q\left(e^{i \theta}\right)=0$ for all $\theta$ on $[0,2 \pi)$ with one exception but it is not so that $\operatorname{Re} q(z) \geqslant 0$ for $|z|<1$. The function $q(z)=[(1+z) /(1-z)]^{5}$ shows that Lemma 3 needs some restriction such as $|\arg q(z)|<\pi$.

THEOREM 1. $P^{*}=P^{-}$.

Proof. Suppose that $p \in P^{-}$. Since $p \in P_{R}$, in order to show that $p \in P^{*}$ we need only show that $q \in P$ where $q(z)=(1+z) p(z) /(1-z)$. This is a consequence of the more general fact that if $q_{1} \in P^{+}$and $p \in P^{-}$then $q_{1} p \in P$. Namely, equations (12) and (13) imply that $\operatorname{Re} q_{1}(z) p(z)=\operatorname{Re} q_{1}(z) \operatorname{Re} p(z)-$ $\operatorname{Im} q_{1}(z) \operatorname{Im} p(z)>0$ for $|z|<1$. Since the function $q_{1}(z)=(1+z) /(1-z)$ belongs to $P^{+}$, we conclude that $P^{-} \subset P^{*}$.

Conversely, suppose that $p \in P^{*}$. Let $z=x+i y$ and $p(z)=u(z)+i v(z)$ and therefore, in particular, $u(z)>0$. Then, equation (9) and the definition of $P^{*}$ imply that

$$
\operatorname{Re} q(x)=\frac{\left[1-\left(x^{2}+y^{2}\right)\right] u(z)-2 y v(z)}{(1-x)^{2}+y^{2}}>0 \text { for }|z|<1 .
$$

Thus, $y v(z)<\frac{1}{2}\left[1-|z|^{2}\right] u(z)$ for $|z|<1$ and consequently $\operatorname{Im} e^{i \theta} \operatorname{Im} p\left(e^{i \theta}\right) \leqslant 0$ for almost all $\theta$. According to Lemma 3 with $q(z)=p(-z)$ we conclude that $\operatorname{Im} z \operatorname{Im} p(z) \leqslant 0$ for $|z|<1$. Therefore, $p \in P^{-}$and so $P^{*} \subset P^{-}$. 
TheOREM 2. A function $q$ in $P^{+}$is an extreme point of $P^{+}$if and only if $q\left(e^{i \theta}\right)$ is real (and nonnegative) or purely imaginary for almost all $\theta$.

Proof. Suppose that $q \in P^{+}$and $q\left(e^{i \theta}\right)$ is real or purely imaginary for almost all $\theta$. Suppose that $q \pm h \in P^{+}$. Since $P^{+}$is convex in order to show that $q$ is an extreme point we need only show that $h$ is the constant function $h=0$.

If $|z|<1$ and $\operatorname{Im} z \geqslant 0$ then $\operatorname{Re}[q(z) \pm h(z)] \geqslant 0$ and $\operatorname{Im}[q(z) \pm h(z)] \geqslant 0$ and so $|\operatorname{Re} h(z)| \leqslant \operatorname{Re} q(z)$ and $|\operatorname{Im} h(z)| \leqslant \operatorname{Im} q(z)$. If $|z|<1$ and $\operatorname{Im} z<0$ then $\operatorname{Re}[q(z) \pm h(z)] \geqslant 0$ and $\operatorname{Im}[q(z) \pm h(z)] \leqslant 0$ and therefore $|\operatorname{Re} h(z)| \leqslant \operatorname{Re} q(z)$ and $|\operatorname{Im} h(z)| \leqslant-\operatorname{Im} q(z)$. Therefore, $|h(z)| \leqslant|q(z)|$ for $|z|<1$ and thus the function $k=h / q$ is analytic in $\Delta,|k(z)| \leqslant 1$ for $|z|<1$ and $k(0)=0$.

The three functions $q, h$ and $k$ all have radial limits on some set of measure $2 \pi$. If $q\left(e^{i \theta}\right)$ is real then $h\left(e^{i \theta}\right)$ is real, because $\operatorname{Im}\left[q\left(e^{i \theta}\right) \pm h\left(e^{i \theta}\right)\right]>0$ when $0<\theta<\pi$ and $\operatorname{Im}\left[q\left(e^{i \theta}\right) \pm h\left(e^{i \theta}\right)\right] \leqslant 0$ when $\pi \leqslant \theta \leqslant 2 \pi$. Thus, $k\left(e^{i \theta}\right)$ is real when $h\left(e^{i \theta}\right)$ is real. If $q\left(e^{i \theta}\right)$ is purely imaginary, then $h\left(e^{i \theta}\right)$ is purely imaginary because $\operatorname{Re}\left[q\left(e^{i \theta}\right) \pm h\left(e^{i \theta}\right)\right] \geqslant 0$, and again $k\left(e^{i \theta}\right)$ is real. Therefore, $k\left(e^{i \theta}\right)$ is real for almost all $\theta$. As $k$ is bounded this implies that $k$ is constant, that is $k=0$. Therefore $h=0$ and $q$ is an extreme point of $P^{+}$.

The converse shall be proved in its contrapositive form. Let $\Lambda$ denote the set consisting of the purely imaginary numbers and the positive real numbers. Suppose that $q \in P^{+}$and that the set $A=\left\{\theta: q\left(e^{i \theta}\right) \notin \Lambda\right\}$ has a positive measure. We shall construct a nonconstant function $h$ so that $q \pm h \in P^{+}$.

Let $\Lambda_{n}^{\prime}=\{w: \operatorname{Re} w \geqslant 1 / n, \operatorname{Im} w \geqslant 1 / n,|w| \leqslant n\} \quad(n=1,2,3, \ldots)$ and let $\Lambda_{n}^{\prime \prime}=\left\{w: \bar{w} \in \Lambda_{n}^{\prime}\right\}$ so that $\Lambda_{n}=\Lambda_{n}^{\prime} \cup \Lambda_{n}^{\prime \prime}$ provides an exhaustion of the set of complex numbers in the (open) first and fourth quadrants with $\Lambda_{n}$ compact and symmetric about the real axis. Let $A_{n}=\left\{\theta: \theta \in A\right.$ and $\left.q\left(e^{i \theta}\right) \in \Lambda_{n}\right\} \quad(n=$ $1,2,3, \ldots)$. Since $\left\{A_{n}\right\}$ is an increasing sequence and $\cup_{n=1}^{\infty} A_{n}=A$, there is an integer $n$ so that $A_{n}$ has positive measure. There is a closed subset of $A_{n}$, denoted $B$, so that $m(B)>0$ and if $\theta \in B$ then $q\left(e^{i \theta}\right)$ exists and belongs to a compact set having a positive distance denoted $\delta$ from $\Lambda$. We also may assume that $m(B)<2 \pi$ as we could replace $B$ by either $B \cap[[0, \pi / 2] \cup[3 \pi / 2,2 \pi]]$ or $B \cap[\pi / 2,3 \pi / 2]$. Since $q(\bar{z})=\overline{q(z)}$ we also may assume that the set $B_{1}=\left\{z: z=e^{i \theta}, \theta \in B\right\}$ is symmetric with respect to the real axis. Let $C_{1}=B_{1} \cap\{w:|w|=1, \operatorname{Im} w>0\}$ and $D_{1}=B_{1} \cap\{w:|w|=1, \operatorname{Im} w \leqslant 0\}$ and let $C$ and $D$ denote the corresponding subsets of $[0,2 \pi]$.

Let $u_{1}$ denote the harmonic measure of $C_{1}$, let $u_{2}$ denote the harmonic measure of $D_{1}$ and let $u=u_{1}-u_{2}$. Then $u$ is harmonic in $\Delta$ and $-1<u(z)<1$ for $|z|<1$. Since $C_{1}$ and $D_{1}$ are conjugate sets, $m(C)=m(D)$. Therefore,

$$
\begin{aligned}
u(0) & =\frac{1}{2 \pi} \int_{0}^{2 \pi} u\left(e^{i \theta}\right) d \theta=\frac{1}{2 \pi}\left\{\int_{0}^{2 \pi} u_{1}\left(e^{i \theta}\right) d \theta-\int_{0}^{2 \pi} u_{2}\left(e^{i \theta}\right) d \theta\right\} \\
& =\frac{1}{2 \pi}\{m(C)-m(D)\}=0
\end{aligned}
$$

Let $v$ denote the harmonic conjugate of $u$ with $v(0)=0$ and let $\omega=v+i u$. The function $\zeta$ defined by $\zeta(z)=\omega(z)+\overline{\omega(\bar{z})}$ is analytic in $\Delta$ and $\zeta(z)$ is real when $z$ is 
real $(|z|<1)$. Suppose that $\theta \notin C \cup D$ and $u_{1}\left(e^{i \theta}\right)$ and $u_{2}\left(e^{i \theta}\right)$ exist. Then $u_{1}\left(e^{i \theta}\right)$ $=u_{2}\left(e^{i \theta}\right)=0$ and so $u\left(e^{i \theta}\right)$ exists and equals 0 . Since $C_{1} \cup D_{1}$ is symmetric $e^{-i \theta} \notin C_{1} \cup D_{1}$ and so $u_{1}\left(e^{-i \theta}\right)=u_{2}\left(e^{-i \theta}\right)=0$ and again $u\left(e^{-i \theta}\right)=0$. As $u_{1}$ and $u_{2}$ have radial limits almost everywhere this proves that $\zeta\left(e^{i \theta}\right)$ exists and is real on a subset $E$ of the complement of $C \cup D$ for which $m(E)=2 \pi-m(C \cup D)$.

Since $-1<u(z)<1$ for $|z|<1$, we conclude that $-2<\operatorname{Im} \zeta(z)<2$ for $|z|<1$. Thus, the function $\sigma=(\exp (\pi \zeta / 4)-1) /(\exp (\pi \zeta / 4)+1)$ is analytic in $\Delta, \sigma(0)=0$, $|\sigma(z)|<1$ for $|z|<1$ and $\sigma(z)$ is real when $z$ is real $(|z|<1)$. Also, if $\theta \in E$ then $\sigma\left(e^{i \theta}\right)$ exists and is real, where if necessary the subset of $E$ (of measure 0 ) on which $\zeta\left(e^{i \theta}\right)=-2$ is deleted.

Let $\varepsilon$ be a real number so that $0<\varepsilon<\min (1, \delta / n)$ and let $h=\varepsilon \sigma q$. Suppose that $\theta \in E$ and $q\left(e^{i \theta}\right)$ exists. Then $\sigma\left(e^{i \theta}\right)$ exists and $\sigma\left(e^{i \theta}\right)$ is real. Therefore, $q\left(e^{i \theta}\right) \pm h\left(e^{i \theta}\right)=\left(1 \pm \varepsilon \sigma\left(e^{i \theta}\right)\right) q\left(e^{i \theta}\right)$, and so $q\left(e^{i \theta}\right) \pm h\left(e^{i \theta}\right)$ is a positive real multiple of $q\left(e^{i \theta}\right)$. In particular, this implies that $q\left(e^{i \theta}\right) \pm h\left(e^{i \theta}\right)$ belongs to the (closed) first or fourth quadrant whenever $q\left(e^{i \theta}\right)$ belongs to that quadrant. Now suppose that $\theta \in(C \cup D) \cap F$ where $F$ is the set (of measure $2 \pi$ ) on which $\sigma\left(e^{i \theta}\right)$ exists. Since $0<\varepsilon<\delta / n,\left|\sigma\left(e^{i \theta}\right)\right|<1$ and $\left|q\left(e^{i \theta}\right)\right| \leqslant n$ it follows that $q\left(e^{i \theta}\right) \pm h\left(e^{i \theta}\right)$ is in the same quadrant as the number $q\left(e^{i \theta}\right)$. Also, we note that $|\arg (q(z) \pm h(z))|<\pi$ for $|z|<1$. This is a consequence of $|\arg q(z)|<\pi / 2$ and $|\arg (1 \pm \varepsilon \sigma(z))|<\pi / 2$, as $\varepsilon<1$ and $|\sigma(z)|<1$.

We have shown that the functions $q+h$ and $q-h$ satisfy the conditions of Lemma 2 and therefore $\operatorname{Re}[q(z) \pm h(z)] \geqslant 0$ for $|z|<1$. The normalizations $q(0)$ $=1$ and $h(0)=0$ imply that $\operatorname{Re}[q(z) \pm h(z)]>0$ for $|z|<1$, that is, $q \pm h \in P$. Also, $q+h$ and $q-h$ satisfy the conditions of Lemma 3 and this yields the conclusions that $q \pm h \in P^{+}$. Since $h$ is not the zero function this completes the proof that $q$ is not an extreme point of $P^{+}$.

Corollary 1. A function $p$ in $P^{-}$is an extreme point of $P^{-}$if and only if $p\left(e^{i \theta}\right)$ is real (and nonnegative) or purely imaginary for almost all $\theta$.

Proof. This result is equivalent to Theorem 2 because the mapping defined by $q(z)=p(-z)$ provides a linear homeomorphism between $P^{-}$and $P^{+}$.

Corollary 2. A function $p$ in $P^{*}$ is an extreme point of $P^{*}$ if and only if $p\left(e^{i \theta}\right)$ is real (and nonnegative) or purely imaginary for almost all $\theta$.

Proof. This result follows from Corollary 1 and the fact that $P^{*}=P^{-}$as proved in Theorem 1.

\section{The extreme points of $Q$.}

THEOREM 3. A function $f$ in $Q$ is an extreme point of $Q$ if and only if $f\left(e^{i \theta}\right)$ is real or purely imaginary for almost all $\theta$.

Proof. Because of Lemma 1 the family $Q$ consists of the functions given by $f(z)=z p\left(z^{2}\right) /\left(1-z^{2}\right)$ where $p$ varies in $P^{*}$. This correspondence between $Q$ and $P^{*}$ is a linear homeomorphism and so extreme points of $Q$ correspond to extreme points of $P^{*}$. If $|z|=1$ and $z \neq \pm 1$ then $z /\left(1-z^{2}\right)$ is purely imaginary. It follows 
that the condition that $p\left(e^{i \theta}\right)$ is real or purely imaginary for almost all $\theta$ is equivalent to the condition that $f\left(e^{i \theta}\right)$ is real or purely imaginary (pointwise, in reverse order) for almost all $\theta$. Therefore, Corollary 2 implies this theorem.

We shall determine the univalent functions in $Q$ which are extreme points of $Q$. Our argument uses the following lemma which may be of more general interest. We recall the fact that if $f$ is univalent in $\Delta$ then the radial limit function $f\left(e^{i \theta}\right)$ exists (and is finite) for almost all $\theta$. In the lemma and later on we use the notation $\partial A$ for the boundary of a set $A, \bar{A}$ for the closure of $A$ and $A^{\prime}$ for the complement of $A$, where the topology is the usual one for the (unextended) complex plane and $A$ is any set of complex numbers.

Lemma 4. Suppose that the function $f$ is analytic and univalent in $\Delta$ and that $\Gamma$ is $a$ measurable subset of $[0,2 \pi]$ with $m(\Gamma)=2 \pi$ so that $f\left(e^{i \theta}\right)$ exists (and is finite) when $\theta \in \Gamma$. If $D=\left\{w: w=f\left(e^{i \theta}\right), \theta \in \Gamma\right\}$ and $F=f(\Delta)$ then $\partial F \subset \bar{D}$.

Proof. Suppose that there is a complex number $w_{0}$ such that $w_{0} \in \partial F$ and $w_{0} \notin \bar{D}$. Since $w_{0} \in \partial F$ it follows that $f(z) \neq w_{0}$ for all $z$ in $\Delta$ because of the openness of the mapping $f$. Therefore, the function $g$ defined by

$$
g(z)=1 /\left(f(z)-w_{0}\right)
$$

is analytic and univalent in $\Delta$. Since $g$ does not vanish the function $h=g^{1 / 3}$ is also analytic and univalent in $\Delta$. The distortion theorem implies that there is a constant $K$ (depending on $g(0)$ and $g^{\prime}(0)$ ) so that $|g(z)| \leqslant K /(1-|z|)^{2}$ for $|z|<1$. Thus, there is a constant $L$ so that $|h(z)| \leqslant L /(1-|z|)^{2 / 3}$ for $|z|<1$. Since the exponent in this estimate is less than 1, Prawitz's inequality [6, p. 127] shows that the integral $(1 / 2 \pi) \int_{0}^{2 \pi}\left|h\left(r e^{i \theta}\right)\right| d \theta$ is a bounded function of $r$ on the interval $(0,1)$. In other words, $h$ belongs to the Hardy class $H^{1}$.

We shall show that if $\theta \in \Gamma$ then $h\left(e^{i \theta}\right)$ exists (and is finite). First notice that since $w_{0} \notin \bar{D}, g\left(e^{i \theta}\right)$ exists whenever $f\left(e^{i \theta}\right)$ exists. In addition, the mapping $\sigma=$ $\zeta^{1 / 3}$ has the property that if $\zeta_{0} \in \partial g(\Delta)$ and if $\zeta$ approaches $\zeta_{0}$ along a curve in $g(\Delta)$ then $\sigma$ has a limit. When $\zeta_{0}=0$ this is a consequence of the relation $\left|\zeta^{1 / 3}\right|=|\zeta|^{1 / 3}$. When $\zeta_{0} \neq 0$ we appeal to the corresponding property of the logarithm function, which is due to its representation as an integral of the analytic function $s=1 / \zeta$ along a path in the simply connected domain $g(\Delta)$ from a fixed point to a variable point.

Since $h \in H^{1}, h$ may be represented as the Poisson integral of its boundary value function $h\left(e^{i \theta}\right)$. This integration may be regarded as taking place only over the set $\Gamma$. Therefore, for each $z$ in $\Delta h(z)$ is a (positively weighted) average of numbers in the set $C=\left\{h\left(e^{i \theta}\right): \theta \in \Gamma\right\}$, and so $h(\Delta)$ is contained in the closed convex hull of $C$. The set $C$ is bounded, since $w_{0} \notin \bar{D}$ and $\lim _{w \rightarrow \infty}\left(1 / w^{1 / 3}\right)=0$. Consequently, $h(\Delta)$ is bounded. On the other hand, because $w_{0} \in \partial F$ there is a sequence $\left\{z_{n}\right\}$ so that $\left|z_{n}\right|<1$ and $f\left(z_{n}\right) \rightarrow w_{0}$. This implies that $\left|g\left(z_{n}\right)\right| \rightarrow \infty$ and $\left|h\left(z_{n}\right)\right| \rightarrow \infty$. This contradicts the boundedness of $h(\Delta)$ and completes the proof.

Lemma 4 is not generally valid without the assumption that $f$ is univalent in $\Delta$. For example, if $f(z)=\exp [-(1+z) /(1-z)]$ and $\Gamma=(0,2 \pi)$ then $D=\{w:|w|=$ $1\}$ and $F=D \cup\{0\}$. A modification of this example is possible so that $\Gamma, D$ and 
$F$ remain the same and $\lim _{r \rightarrow 1} f(r)$ does not exist.

THEOREM 4. The set of functions in $Q$ that are univalent in $\Delta$ and are extreme points of $Q$ is given by

$$
f(z)=\frac{z}{\left[\left(1-x z^{2}\right)\left(1-\bar{x} z^{2}\right)\right]^{1 / 2}} \quad(|x|=1) .
$$

Proof. We shall give a characterization of the set given by equation (14) in terms of the mapping properties of these functions. We first consider the related set of functions

$$
g(z)=\frac{z}{(1-x z)(1-\bar{x} z)} \quad(|x|=1) .
$$

Each function $g$ is analytic and univalent in $\Delta$, and $g(\Delta)$ consists of the complex plane excluding the slits $\left[-\infty,-1 /|1+x|^{2}\right]$ and $\left[1 /|1-x|^{2}, \infty\right]$ on the real axis. We claim that there are no other functions than those given by equation (15) that have the properties: $g$ is analytic and univalent in $\Delta ; g(0)=0$ and $g^{\prime}(0)=1 ; g(z)$ is real when $z$ is real and the only excluded values of $g$ are real. To see this result, we first note that any such function has the property that if $g(z) \neq a$ with $a>0$ then $g(z) \neq b$ for $b>a$. Likewise if $g(z) \neq-a$ with $a>0$ then $g(z) \neq-b$ with $b>a$. Thus, the excluded values of $g$ consist of one or two (unbounded) intervals on the real axis. Now, if $g_{1}$ and $g_{2}$ are two functions having the properties prescribed above and if $g_{1}$ excludes the intervals $[-\infty,-a]$ and $\left[b_{1}, \infty\right]$ and if $g_{2}$ excludes the intervals $[-\infty,-a]$ and $\left[b_{2}, \infty\right]$, where $a, b_{1}$ and $b_{2}$ are real and positive, then $b_{1}=b_{2}$. This follows from the principle of subordination, namely, if, say, $b_{1}<b_{2}$ then $g_{1}$ is subordinate to $g_{2}$ and so $\left|g_{1}^{\prime}(0)\right|<\left|g_{2}^{\prime}(0)\right|$ as equality in the general inequality $\left|g_{1}^{\prime}(0)\right| \leqslant\left|g_{2}^{\prime}(0)\right|$ can occur only when $g_{1}$ and $g_{2}$ have the same range. This contradicts the normalizations $g_{1}^{\prime}(0)=g_{2}^{\prime}(0)=1$, and so $b_{1}=b_{2}$. A similar argument shows that if two functions with the prescribed properties exclude the intervals $\left[-\infty,-a_{1}\right],[b, \infty]$ and $\left[-\infty, a_{2}\right],[b, \infty]$, respectively, with $a_{1}, a_{2}$ and $b$ positive, then $a_{1}=a_{2}$. In other words, there is at most one pair $(a, b)$ so that a function $g$ (with the given properties) has $[-\infty,-a]$ and $[b, \infty]$ as excluded values. Because of the Koebe covering theorem, $b$ is restricted by $b \geqslant 1 / 4$. As $x$ varies on the circle $|x|=1$ the number $1 /\left(|1-x|^{2}\right)$ takes on all values $b$ with $b>1 / 4$.

The set of normalized, real, univalent functions are in one-to-one correspondence with the set of normalized, real, odd univalent functions through the relation

$$
f(z)=\sqrt{g\left(z^{2}\right)} .
$$

The set given by equation (15) corresponds to the set given by equation (14) through the mapping (16). Therefore, the observations in the previous paragraph imply that the set of functions given by equation (14) consist of exactly those functions that are analytic and univalent in $\Delta$, satisfy $f(0)=0, f^{\prime}(0)=1, f(z)$ is real when $z$ is real, $f$ is odd and only have real or purely imaginary numbers for excluded values. 
The functions given by equation (14) belong to $Q$ since they are real, odd and univalent in $\Delta$. They are extreme points of $Q$ because of .' heorem 3 , for $f(z)$ is real or purely imaginary whenever $|z|=1$ with either two or four exceptional values (where $f=\infty$ ).

Conversely, suppose that $f \in Q, f$ is an extreme point of $Q$ and $f$ is univalent in $\Delta$. Theorem 3 implies that $f\left(e^{i \theta}\right)$ is real or purely imaginary for almost all $\theta$. Thus, because of Lemma 4, the boundary of the set of excluded values of $f$ is a subset of the real and imaginary axes. Consequently, $f$ must take on all values off the axes. As we have just shown, this determines that $f$ must have the form of equation (14).

5. Further symmetric families. Let $k$ be a positive integer and define the sectors $A_{j}$ and $B_{j}$ by

$$
A_{j}=\{w: 2(j-1) \pi / k<\arg w<2 j \pi / k\} \quad \text { and } \quad B_{j}=\left\{\begin{array}{r}
\left.-i \pi / k w: w \in A_{j}\right\} \\
\text { for } j=1,2, \ldots, k .
\end{array}\right.
$$

We shall introduce three classes of functions denoted by $T_{k}, \tilde{T}_{k}$ and $Q_{k}$. A function in each class is assumed to be analytic in $\Delta$ and normalized by the conditions $f(0)=0$ and $f^{\prime}(0)=1$. The following conditions define the classes.

(A) $f \in T_{k}$ if $f(z) \in \bar{A}_{j}$ whenever $z \in \bar{A}_{j} \cap \Delta(j=1,2, \ldots, k)$,

(B) $f \in \tilde{T}_{k}$ if $f(z) \in \bar{B}_{j}$ whenever $z \in \bar{B}_{j} \cap \Delta(j=1,2, \ldots, k)$,

(C) $Q_{k}=T_{k} \cap \tilde{T}_{k}$.

The classes $T_{k}, \tilde{T}_{k}$ and $Q_{k}$ relate to families already discussed in this paper. For example, $T_{2}=T$ and $Q_{2}=Q$. An interesting subclass of $Q_{k}$ is the set of univalent functions that are real and $k$-fold symmetric. This relation is not difficult to show. We recall that a function is called $k$-fold symmetric if its power series has the form

$$
f(z)=\sum_{n=0}^{\infty} a_{n k+1} z^{n k+1} \quad(|z|<1) .
$$

This condition on the power series is equivalent to the condition that $f\left(e^{2 \pi i / k} z\right)=$ $e^{2 \pi i / k} f(z)$ for $|z|<1$, as is easily verified. An important example is the function defined by

$$
f(z)=\frac{z}{\left(1-z^{k}\right)^{2 / k}} \quad(|z|<1) .
$$

This function is univalent in $\Delta$ and $f(\Delta)$ is the complement of the slits $\left\{w: w=r e^{i \theta}\right.$, $\left.r \geqslant(1 / 4)^{1 / k}, \theta=\pi / k+(j-1) 2 \pi / k\right\}(j=1,2, \ldots, k)$.

From the definitions of $T_{k}$ and $\tilde{T}_{k}$ it follows that if $f \in T_{k}$ then the function $g$ defined by $g(z)=e^{-i \pi / k} f\left(e^{i \pi / k} z\right)$ belongs to $\tilde{T}_{k}$. Conversely, if $g \in \tilde{T}_{k}$ then $f \in T_{k}$. Also, if $f \in T_{k}$ then the coefficients of $f$ must be real, because $f(z)$ is real (and nonnegative) when $z$ is real and nonnegative. If $k$ is odd and $g \in \tilde{T}_{k}$ then $g$ has real coefficients since $g(z) \leqslant 0$ when $z \leqslant 0(|z|<1)$. When $k$ is even and $g \in \tilde{T}_{k}$ the coefficients are not necessarily real.

LEMMA 5. (a) If $k$ is odd and $f$ belongs to $T_{k}$, then $f$ is $k$-fold symmetric.

(b) If $k$ is even $(k \neq 2)$ and $f$ belongs to $T_{k}$, then $f$ is $(k / 2)$-fold symmetric.

(c) If $f \in Q_{k}$ then $f$ is $k$-fold symmetric. 
Proof. Suppose that $k$ is odd and $f \in T_{k}$. Let $g$ be defined by $g(z)=f(z)-$ $e^{-2 \pi i / k} f\left(e^{2 \pi i / k} z\right)$. If we let $z=r e^{i \theta}$ where $0 \leqslant r<1$ and $\theta=(k-1) \pi / k$ then, as $f$ has real coefficients, we find that

$$
g\left(r e^{i \theta}\right)=f\left(r e^{i \theta}\right)-e^{-2 \pi i / k} f\left(r e^{(k+1) \pi i / k}\right)=f\left(r e^{i \theta}\right)-e^{-2 \pi i / k} \overline{f\left(r e^{(k-1) \pi i / k}\right)}=0 .
$$

Therefore, $g(z)=0$ for $|z|<1$. This is the same as the condition that $f$ be $k$-fold symmetric.

Now suppose that $k$ is even and $f \in T_{k}$. Let $g$ be defined by $g(z)=f(z)-$ $e^{-4 \pi i / k} f\left(e^{4 \pi i / k} z\right)$. If we let $z=r e^{i \theta}$ where $0<r<1$ and $\theta=(k-2) \pi / k$ we find that $g(z)=0$ because $f$ has real coefficients. Thus, $g(z)=0$ for $|z|<1$, which is the same as the condition that $f$ be $(k / 2)$-fold symmetric.

When $k$ is odd, part (c) follows from part (a) since $Q_{k} \subset T_{k}$. Now, suppose that $f \in Q_{k}$ and $k$ is even. Since $f \in T_{k}, f$ is real. Also, by part (b) $f$ is $(k / 2)$-fold symmetric and so we may write

$$
f(z)=\sum_{j=0}^{\infty} a_{m j+1} z^{m j+1} \quad(|z|<1) \text { with } m=k / 2 .
$$

Since $f \in \tilde{T}_{k}$ the function $g$ defined by $g(z)=e^{\pi i / k} f\left(e^{-\pi i / k} z\right)$ belongs to $T_{k}$. The series for $g$ takes on the form

$$
g(z) \sum_{j=0}^{\infty} a_{m j+1} e^{-\pi j i / 2} z^{m j+1}
$$

and as $g \in T_{k}$ all of the coefficients must be real. Since $a_{m j+1}$ are real this implies that $a_{m j+1}=0$ for every odd value of $j$. This proves that $f$ is $k$-fold symmetric.

Definition. Let $P_{k}$ denote the set of functions $p$ that are analytic and nonvanishing in $\Delta$ and satisfy $p(0)=1, p(z)$ is real when $z$ is real and $|\arg p(z)|<\pi / k$ for $|z|<1$.

This definition only requires that $k>0$ but our interest is in the cases $k=$ $2,3,4, \ldots$ We note that $P_{2}=P_{R}$.

LEMMA 6. (a) $f \in T_{k}$ if and only if there is a function $p$ belonging to $P_{k}$ so that

$$
f(z)=\frac{z}{\left(1-z^{k}\right)^{2 / k}} p\left(z^{k}\right)
$$

when $k$ is odd, or

$$
f(z)=\frac{z}{\left(1-z^{k}\right)^{2 / k}} p\left(z^{k / 2}\right)
$$

when $k$ is even.

(b) $f \in \tilde{T}_{k}$ if and only if there is a function $q$ belonging to $P_{k}$ so that

$$
f(z)=\frac{z}{\left(1+z^{k}\right)^{2 / k}} q\left(z^{k}\right)
$$

when $k$ is odd, or

$$
f(z)=\frac{z}{\left(1+z^{k}\right)^{2 / k}} q\left(i z^{k / 2}\right)
$$

when $k$ is even. 
Proof. Suppose that $f \in T_{k}$. Let the functions $q_{r}$ be defined by $q_{r}(z)=$ $\left(1-z^{k}\right)^{2 / k} f(r z) / z$ for each $r$ on $(0,1)$. Let $W_{k}=\{w$ : $|\arg w|<\pi / k\}$. The condition that $f(z) \in \bar{A}_{j}$ when $z \in \bar{A}_{j} \cap \Delta$ and the mapping properties of the function in equation (18) imply that if $|z|=1$ then $q_{r}(z) \in \bar{W}_{k}$. We may apply the Poisson integral formula to $q_{r}$ and this implies that $q_{r}(z) \in \bar{W}_{k}$ for $|z|<1$ due to the convexity of $\bar{W}_{k}$. Letting $r \rightarrow 1$ we conclude that $q(z) \in \bar{W}_{k}$ for $|z|<1$, where the function $q$ is defined by $q(z)=\left(1-z^{k}\right)^{2 / k} f(z) / z$. The normalization $q(0)=f^{\prime}(0)$ $=1$ implies that $q(z) \in W_{k}$ for $|z|<1$ and, therefore, $q \in P_{k}$. Depending on the parity of $k$ we may appeal to part (a) or (b) of Lemma 5 in order to derive equation (19) or (20) with $g(z)=p\left(z^{k}\right)$ or $q(z)=p\left(z^{k / 2}\right)$. This proves one-half of part (a) of this lemma.

In order to prove the converse statement, first consider the case $k$ is odd and assume that equation (19) defines $f$ where $p \in P_{k}$. Let $q$ be defined by $q(z)=$ $p\left(z^{k}\right)$. We may assume that $q$ is analytic in $\bar{\Delta}$, since the general result would be deduced from that for $q(r z)$ with $0<r<1$ by letting $r \rightarrow 1$. The function $f$ satisfies $f(x) \geqslant 0$ for $0 \leqslant x<1, f\left(e^{2 \pi i / k} x\right)=y e^{2 \pi i / k}$ where $y \geqslant 0$ whenever $0 \leqslant x<1$ and $f(z) \in \bar{A}_{1}$ when $|z|=1$ and $0<\arg z<2 \pi / k$. We may write

$$
f(z)=q(z) /(1-z)^{2 / k}
$$

where $g$ is analytic at $z=1$ and $g(z)>0$ for $0 \leqslant z \leqslant 1$. We shall show that this implies that if $N$ is a sufficiently small neighborhood of 1 then $f\left(N \cap \Delta \cap \bar{A}_{1}\right) \subset$ $\bar{A}_{1}$. By taking a suitable power of $f$ in order to introduce a simple pole at $z=1$, this assertion follows from the local behavior of analytic functions expressed at $z=0$ as follows. If $h$ is analytic at $z=0, h$ has a simple zero at $z=0$ and if $h(z)$ is real and nonnegative when $z \geqslant 0$ and $z$ is small, then to each given $\varepsilon(\varepsilon>0)$ and $\theta_{0}$ $\left(0<\theta_{0}<2 \pi\right)$ there exists an $r_{0}\left(r_{0}>0\right)$ so that if $0<\arg z<\theta_{0}$ and $|z|<r_{0}$ then $0<\arg h(z)<\theta_{0}+\varepsilon$. We merely need to observe that $\arg h\left(r e^{i \theta}\right)$ is an increasing function of $\theta$ since $h$ is locally starlike with respect to the origin, and $\arg h\left(r e^{i \theta_{0}}\right)$ is as close to $\theta_{0}$ as we like if $r_{0}$ is near 0 . The application of these relations here needs only $\theta_{0}=\pi / 2$ and $\varepsilon=\pi / 2$. The local behavior of $f$ at the point $z_{0}=e^{i \theta}, \theta=$ $2 \pi / k$ similarly implies that if $N_{1}$ is a sufficiently small neighborhood of $z_{0}$ then $f\left(N_{1} \cap \Delta \cap \overline{A_{1}}\right) \subset \overline{A_{1}}$. The several properties of $f$ in $\bar{A}_{1}$ imply that $f\left(\overline{A_{1}} \cap \Delta\right) \subset \overline{A_{1}}$. (Our argument here is an extension of the one given by Rogosinski in [8] in order to obtain the corresponding fact about $T$.) A similar argument applies to each sector $A_{j}$ in order to deduce that $f\left(\overline{A_{j}} \cap \Delta\right) \subset \overline{A_{j}}$ for $j=1,2, \ldots, k$. This completes the proof that $f \in T_{k}$. A similar argument applies when $k$ is even, as a consequence of equation (20).

Part (b) of this lemma is a consequence of part (a) and the fact that $f \in \tilde{T}_{k}$ if and only if we may write $f(z)=e^{-\pi / k} g\left(e^{i \pi / k} z\right)$ where $g \in T_{k}$. The form of equation (21) occurs because $q \in P_{k}$ when $q(z)=p(-z)$ and $p \in P_{k}$.

Lemma 7. $f \in Q_{k}$ if and only if there is a function $p$ belonging to $P_{k}$ so that

$$
f(z)=\frac{z}{\left(1-z^{k}\right)^{2 / k}} p\left(z^{k}\right)
$$


and

$$
f(z)=\frac{z}{\left(1+z^{k}\right)^{2 / k}} q\left(z^{k}\right) .
$$

Proof. When $k$ is odd this lemma is an immediate consequence of Lemma 6 since $Q_{k}=T_{k} \cap \tilde{T}_{k}$. When $k$ is even we additionally need to appeal to part (c) of Lemma 5 .

THEOREM 5. $f \in Q_{k}$ if and only if there is a function $p$ in $P_{k}$ so that equation (23) holds and $p$ has the property $\operatorname{Im} p(z) \cdot \operatorname{Im} z \leqslant 0$ for $|z|<1$.

Proof. Suppose that $f \in Q_{k}$. Lemma 7 implies the existence of two functions $p$ and $q$ so that equations (23) and (24) hold. This implies that

$$
q\left(z^{k}\right) / p\left(z^{k}\right)=\left(\left(1+z^{k}\right) /\left(1-z^{k}\right)\right)^{2 / k}
$$

which may be expressed as $q_{1}(z) / p_{1}(z)=(1+z) /(1-z)$, where $q_{1}=q^{k / 2}$ and $p_{1}=p^{k / 2}$. Since $q$ and $p$ belong to $P_{k}$ it follows that $q_{1}$ and $p_{1}$ belong to $P$. As $f$ is real so is $p_{1}$ and so we see that $p_{1} \in P^{*}$. Because of Theorem 1 this implies that $p_{1} \in P^{-}$. Since the mapping $w=\zeta^{2 / k}$ satisfies $\operatorname{Im} w \cdot \operatorname{Im} \zeta \geqslant 0$ whenever $\operatorname{Re} \zeta>$ 0 we conclude that $p$ has the additional property expressed as $\operatorname{Im} p(z) \cdot \operatorname{Im} z \leqslant 0$ for $|z|<1$.

The converse follows because equations (23) and (24) characterize functions in $Q_{k}$ and since $P^{-}=P^{*}$ we may reverse the several relations.

The relations we have developed in this section enable us to determine the set of extreme points of each family of symmetric functions. The arguments are similar to those given in the initial part of this paper and in some cases are easier, since, for example, the Poisson formula is directly applicable to functions having a range in the set $\{w$ : $|\arg w|<\pi / k\}$ whenever $k>1$. We shall present the result only for the family $Q_{k}$.

THEOREM 6. $A$ function $f$ in $Q_{k}$ is an extreme point of $Q_{k}$ if and only if the radial limit function $f\left(e^{i \theta}\right)$ belongs to one of the rays $\arg w= \pm j \pi / k(j=0,1, \ldots, k)$ for almost all $\theta$ on $[0,2 \pi]$.

Proof. According to Theorem 5, the family $Q_{k}$ is in one-to-one correspondence with the set of functions in $P_{k}$ with the property $\operatorname{Im} p(z) \cdot \operatorname{Im} z<0$, through equation (23). This established a linear homeomorphism between these two classes and so their extreme points are in one-to-one correspondence by the relation (23). The extreme points of this distinguished subset of $P_{k}$ may be characterized through the property that $\arg p\left(e^{i \theta}\right)= \pm \pi / k$ for almost all $\theta$ on $[0,2 \pi]$. The argument uses the techniques given in the proof of Theorem 2 . The property of $p$ transforms to the stated property of $f$ because of equation (23) and the mapping properties of the function $w=z /\left(1-z^{k}\right)^{2 / k}$ on $|z|=1$. 


\section{Coefficient estimates.}

LEMMA 8. Suppose that $\varphi$ is an analytic function which satisfies $|\varphi(z)|<1$ for $|z|<1, \varphi(0)=0$ and $\operatorname{Im} \varphi(z) \cdot \operatorname{Im} z \geqslant 0$ for $|z|<1$. If

$$
\varphi(z)=\sum_{n=1}^{\infty} c_{n} z^{n}
$$

then $0 \leqslant c_{1} \leqslant 1$ and

$$
\left|c_{2}\right| \leqslant 2 c_{1}\left(1-c_{1}\right) \text {. }
$$

Proof. The condition $\operatorname{Im} \varphi(z) \cdot \operatorname{Im} z>0$ implies that the coefficients $\left\{c_{n}\right\}$ are real and, except in the case $\varphi=0$, that $c_{1}>0$. The fact that $|\varphi(z)|<1$ for $|z|<1$ generally implies that $\left|c_{1}\right| \leqslant 1$ and so $0 \leqslant c_{1} \leqslant 1$. In order to prove equation (26) we may assume that $c_{1}>0$. Let the analytic functions $g$ and $h$ be defined by $g(z)=\varphi(z) /(1-\varphi(z))^{2}$ and $h(z)=-\varphi(-z) /(1+\varphi(-z))^{2}$. Since the function $k$ defined by $k(z)=z /(1-z)^{2}$ also satisfies $\operatorname{Im} k(z) \cdot \operatorname{Im} z \geqslant 0$ the same condition holds for $g$ and $h$, being composites of two such functions. If we let $d_{1}=g^{\prime}(0)$ and $e_{1}=h^{\prime}(0)$ then $d_{1}=e_{1}=c_{1}$ and so $d_{1}>0$ and $e_{1}>0$. Hence $g / d_{1}$ and $h / e_{1}$ belong to $T$. Any function $f$ belonging to $T$ with $f(z)=z+a_{2} z^{2}+\ldots$ satisfies $\left|a_{2}\right| \leqslant 2$. Applying this inequality to $g / d_{1}$ and $h / e_{1}$ we find that $\left|2 c_{1}^{2}+c_{2}\right|<2 c_{1}$ and $\left|2 c_{1}^{2}-c_{2}\right| \leqslant 2 c_{1}$, respectively. These two inequalities imply the desired inequalities $-2 c_{1}\left(1-c_{1}\right) \leqslant c_{2} \leqslant 2 c_{1}\left(1-c_{1}\right)$.

We also show that inequality (26) is sharp and find all extremal functions. The condition $c_{2}=2 c_{1}\left(1-c_{1}\right)$ is equivalent to $a_{2}=2$ which occurs only when $g(z) / d_{1}$ $=z /(1-z)^{2}$, that is, where $\varphi$ is determined by $\varphi(z) /(1-\varphi(z))^{2}=c_{1} z /(1-z)^{2}$. This function $\varphi$ maps $\Delta$ one-to-one onto $\Delta$ slit along part of the negative real axis and for any $c_{1}, 0<c_{1}<1$, satisfies $\operatorname{Im} \varphi(z) \cdot \operatorname{Im} z>0$. Likewise, the equality $c_{2}=-2 c_{1}\left(1-c_{1}\right)$ requires that $h(z) / e_{1}=z /(1-z)^{2}$. This is the same as $\varphi(z) /(1+\varphi(z))^{2}=c_{1} z /(1+z)^{2}$, which for any $c_{1}, 0<c_{1}<1$, produces a univalent mapping of $\Delta$ onto $\Delta$ slit along part of the positive real axis.

THEOREM 7. Suppose that $f \in Q_{k}$ and

$$
f(z)=\sum_{n=0}^{\infty} a_{n k+1} z^{n k+1} .
$$

Then

$$
-2 / k \leqslant a_{k+1} \leqslant 2 / k
$$

and

$$
-1 / k \leqslant a_{2 k+1} \leqslant 3 / k
$$

Each inequality is sharp.

Proof. Suppose that $f \in Q_{k}$ and $p$ and $q$ are defined by equations (23) and (24). Write

$$
p(z)=1+p_{1} z+p_{2} z^{2}+\ldots
$$


and

$$
q(z)=1+q_{1} z+q_{2} z^{2}+\ldots .
$$

Since $p$ and $q$ belong to $P_{k}$, they are subordinate to the function given by $w=((1+z) /(1-z))^{2 / k}=1+4 z / k+\ldots$ and consequently $\left|p_{1}\right|<4 / k$ and $\left|q_{1}\right| \leqslant 4 / k$. Equating coefficients on both sides of equations (23) and (24) we find that $a_{k+1}=2 / k+p_{1}$ and $a_{k+1}=-2 / k+q_{1}$. Therefore, $\left|a_{k+1}-2 / k\right|<4 / k$ and $\left|a_{k+1}+2 / k\right| \leqslant 4 / k$. Using one-half of each of these inequalities, we obtain inequality (28).

Because of Theorem 5, we may write

$$
p(z)=[(1-\varphi(z)) /(1+\varphi(z))]^{2 / k},
$$

where $\varphi$ satisfies the conditions of Lemma 8. From equations (23) and (32) we find that

$$
a_{2 k+1}=\frac{2}{k^{2}}+\frac{1}{k}-\frac{8}{k^{2}} c_{1}+\frac{8}{k^{2}} c_{1}^{2}-\frac{4}{k} c_{2}
$$

The inequality $c_{2} \geqslant-2 c_{1}\left(1-c_{1}\right)$ implies that

$$
a_{2 k+1} \leqslant 2 / k^{2}+1 / k+\left(8 / k^{2}\right)(k-1)\left(c_{1}-c_{1}^{2}\right)
$$

and as $c_{1}-c_{1}^{2} \leqslant 1 / 4$ we obtain the estimate $a_{2 k+1} \leqslant 3 / k$. Using $c_{2} \leqslant 2 c_{1}\left(1-c_{1}\right)$ and the inequality $c_{1}^{2}-c_{1} \geqslant-1 / 4$ we find that $a_{2 k+1} \geqslant-1 / k$.

The equality $a_{k+1}=-2 / k$ requires that $p_{1}=-4 / k$, which holds only when $p(z)=((1-z) /(1+z))^{2 / k}$. This implies that $f(z)=z /\left(1+z^{k}\right)^{2 / k}$. Similarly, the equality $a_{k+1}=2 / k$ requires that $p_{1}=4 / k$. This implies that

$$
q(z)=((1+z) /(1-z))^{2 / k} \text { and } f(z)=z /\left(1-z^{k}\right)^{2 / k} \text {. }
$$

The equality $a_{2 k+1}=-1 / k$ requires that $c_{2}=2 c_{1}\left(1-c_{1}\right)$ and $c_{1}=1 / 2$. From the remarks after the proof of Lemma 8 this implies that

$$
\varphi(z) /(1-\varphi(z))^{2}=z / 2(1-z)^{2} .
$$

If $p$ is defined by equation (32) then $\operatorname{Im} p(z) \cdot \operatorname{Im} z<0$ because $\operatorname{Im} \varphi(z) \cdot \operatorname{Im} z>$ 0 . Therefore, if $f$ is defined by equation (23) then $f \in Q_{k}$ and it is the only function for which $a_{2 k+1}=-1 / k$. In a similar way the equality $a_{2 k+1}=3 / k$ associates with the conditions $c_{1}=-2 c_{1}\left(1-c_{1}\right)$ and $c_{1}=1 / 2$. This determines $\varphi$ by $\varphi(z) /(1+\varphi(z))^{2}=z / 2(1+z)^{2}$ which in turn determines $p$ and then $f$.

The inequality (28) compares with the result that $\left|a_{k+1}\right|<2 / k$ whenever $f(z)=$ $z+a_{k+1} z^{k+1}+\ldots$ is $k$-fold symmetric and univalent in $\Delta$. For such functions the sharp result [3]

$$
\left|a_{2 k+1}\right| \leqslant(2 / k) \exp [-2(k-1) /(k+1)]+1 / k
$$

is quite different from inequality (29). Even for univalent functions with real coefficients the coefficient estimates are generally more restrictive than that given by Theorem 7. For example, when $f$ is odd and univalent, $\left|a_{5}\right|<e^{-2 / 3}+\frac{1}{2}=$ $1.013 \ldots$, as stated above, but, in addition, there is an odd univalent function with real coefficients for which $a_{5}=e^{-2 / 3}+\frac{1}{2}[9$, p. 633]. 
For the class $Q_{2}$, Robertson [7] proved that $\left|a_{3}\right| \leqslant 1$ and, in general, $\left|a_{2 n-1}\right|+$ $\left|a_{2 n+1}\right| \leqslant 2$. For the family of odd, typically real functions the coefficents are much less restrictive than for $Q_{2}$ and the bound $\left|a_{2 n+1}\right| \leqslant 2 n+1[8]$ is the sharp result. This bound may be viewed as a consequence of the knowledge of the extreme points of this class, as determined by Hallenbeck in [4]. It does not seem that extreme point methods will be useful to solve extremal problems for the classes $Q_{k}$ because of the diversity of extreme points of $Q_{k}$ as given by Theorem 6 . Theorem 4 provides some idea of the distinction between $Q$ and the closed convex hull of the set of odd univalent functions with real coefficients.

ACKNowledgement. We thank J. S. Hwang for several helpful comments about this paper. In particular, the proof of Theorem 1 given here was suggested by Hwang as a simplification of our original argument.

\section{REFERENCES}

1. L. Brickman, T. H. MacGregor and D. R. Wilken, Convex hulls of some classical families of univalent functions, Trans. Amer. Math. Soc. 156 (1971), 91-107.

2. P. L. Duren, Theory of $\boldsymbol{H}^{p}$ spaces, Academic Press, New York, 1970.

3. M. Fekete and G. Szegö, Eine Bemerkung über ungerade schlichte Funktionen, J. London Math. Soc. 8 (1933), 85-89.

4. D. J. Hallenbeck, Convex hulls and extreme points of families of starlike and close-to-convex mappings, Pacific J. Math. 57 (1975), 167-176.

5. J. G. Milcetich, On the extreme points of some sets of analytic functions, Proc. Amer. Math. Soc. 45 (1974), 223-228.

6. Chr. Pommerenke, Univalent functions, Vandenhoeck and Ruprecht, Göttingen, 1975.

7. M. S. Robertson, On the coefficients of a typically-real function, Bull. Amer. Math. Soc. 41 (1935), 565-572.

8. W. Rogosinski, Über positive harmonische Entwicklungen und typisch-reelle Potenzreihen, Math. $\mathrm{Z}$. 35 (1932), 93-121.

9. A. C. Schaeffer and D. C. Spencer, The coefficients of schlicht functions, Duke Math. J. 10 (1943), 611-635.

10. M. Tsuji, Potential theory in modern function theory, Maruzen, Tokyo, 1975.

Department of Mathematics, State University of New York, Center at Albany, Albany, New YORK 12222 